\title{
Planar HTS Device Process Using Ion Implantation
}

\author{
Q. Y. Ma \\ Department of Electrical Engineering, Columbia University \\ New York, NY 10027, USA
}

\begin{abstract}
A planar inhibiting fabrication technique of HTS electronic devices has been developed in recent years and is summarized. A systematic study of the properties of ion inhibited HTS films is presented. The inhibition of superconductivity is carried out by the implantation of reactive ions such as $\mathrm{Al}, \mathrm{B}, \mathrm{Ca}$, and $\mathrm{Si}$ into $\mathrm{YBCO}$ epitaxial films. The inhibited films are characterized using resistivity, susceptibility, SIMS, XRD, XPS, and SEM measurements. The results indicate that the implanted ions react strongly with oxygen, which turn the films resistive, and even insulative without altering the overall crystalline structure of the films. The effect of ion diffusion is also investigated. Ion gettering phenomenon is observed in Si implanted films. Those effects define the pattern resolution of the planar inhibiting fabrication process. The ion implantation process is applied to the fabrication of HTS single layer devices. These devices include Josephson junctions, DC SQUIDs, RF coils, and microwave waveguides. Operational step-edge junctions and DC SQUIDs with a minimum width of 2 $\mu \mathrm{m}$ were formed and tested at $77 \mathrm{~K}$. Passive devices such as low loss waveguides ( $1-25 \mathrm{GHz}$ ) and high $Q$ resonantors $(33 \mathrm{MHz}$ ) were demonstrated. The performance of these devices, in general, is better than or at least equal to that of dry etched devices. However, the new process offers two major advantages. First, the patterned device is planar, which allows a multilayer device to be built, and second, there is little or no chemical contamination of the patterned devices. To demonstrate the viability of this technique for the fabrication of multilayer devices, simple YBCO/STO/YBCO tri-layer structures (such as a crossover and a parallel-plate capacitor) with two implantations were fabricated.
\end{abstract}

\section{INTRODUCTION}

During the past few years, much progress has been made in high temperature superconductor (HTS) electronics. HTS Josephson junctions and SQUIDs with high performance and low noise have been made [1,2]. A HTS multilayer integrated SQUID magnetometer [3] and a multichip module [4] were recently fabricated. However, many devices demonstrated in the $\mathrm{R} \& \mathrm{D}$ laboratories so far have problems with low yield and/or poor stability [5]. This may be attributed to the lack of reliable fabrication technologies with the necessary processing environment for HTS oxide devices.

Manuscript received Aug. 27. 1996.

This work was supported in part by the NSF under grant No. DMR9531208 and ECS-9624654, by the Innovative Enhancement Fund at Columbia university.
Unlike the metallic superconductors, the HTS oxides are extremely sensitive to surface contamination. Exposing the surface of HTS oxides to chemicals, water, or even air can change the material properties from superconducting to insulating [6,7]. Conventional patterning processes involve the removal of material by ion milling or chemical etching [8], which contaminates the surfaces and sidewalls of the patterned structure. Such contamination severely degrades the device performance.

In order to enhance the performance and improve the sensitivity of many superconducting devices, multilayer structures are often required. Multilayer device configurations are also essential for circuit integration and packaging. Examples of HTS multilayer devices include SQUIDs [3], multichip modules [4], and flux transformers [9]. Conventional fabrication processes render the patterned surface no longer flat, which complicates the processing of subsequent layers.

The inhibition of superconductivity in HTS materials by silicon [10-13] was originally developed in early 1989 . The process introduces a reactive element; silicon, into HTS films by intermixing the film with a silicon film or silicon substrate. The intermixing is accomplished by thermal diffusion of the silicon at a high temperature. The silicon atoms then react with oxygen in YBCO and form silicon oxides, transforming the material from superconducting to insulating. Since 1989, this technique has been used to fabricate a variety of HTS electronic devices and components, including current controlled switches [14], submicron weak-links [15], Josephson junctions [16], SQUIDs [17], and microbolometers [18,19]. However, there is one drawback of this method. The intermixing of a silicon film with a HTS film results in an amorphous film, thus limiting the technique to single layer patterning since additional layers can not be epitaxially grown on top of the mixed area.

To overcome this problem, a new method of inhibition by reactive ion implantation (RII) was recently demonstrated $[20,21]$. The RII process maintains the crystallinity of the HTS film while inhibiting the electrical conductivity and diamagnetism. HTS lines of approximately $2-10 \mu \mathrm{m}$ in width have been fabricated by IRII with no degradation of the superconducting properties in the patterned HTS area [20]. The patterned lines exhibited very sharp edges with a resolution of $0.1 \mu \mathrm{m}$ or less. More importantly, a second layer of HTS film was successfully grown epitaxially on the 




Fig.1. X-ray Photoelectron Spectroscopy(XPS) of $\mathrm{Si}_{2 \mathrm{p}}$ of a $\mathrm{Si}$ implanted YBCO film.

patterned film and subsequently patterned with ion implantation. The second layer of film. had the similar material properties (e.g. smoothness, critical temperature and current density) as the base layer. Simple three layer. structures like a vertical contact and a crossover have been demonstrated [21].

For the device processing, several single layer devices have been fabricated using the RII technique. HTS bolometers have been patterned using silicon ion implantation [22]. Other devices include DC-SQUIDs [23], microwave components $[24,25]$ and RF coils [26-28]. The new RII process has several advantages. Firstly, the patterned HTS structure is contained within the film (i.e. the film remains planar), preventing the sidewalls from contamination. Secondly, the process preserves the crystallinity of the HTS material allowing additional layers to be grown epitaxially.

This paper serves as a review for the material study of ion implanted HTS films and as a summary for the planar IRII processing technique of HTS electronic devices.

\section{Material STUdy}

The HTS materials used are YBCO films grown on $\mathrm{LaAlO}_{3}$ or $\mathrm{SrTiO}_{3}$ (100) substrates either by pulsed laser deposition or by sputtering. A typical film is about 1000 $2000 \AA$ thick. It has a $\mathrm{T}_{\mathrm{c}}$ around $85-92 \mathrm{~K}$ and a $\mathrm{J}_{\mathrm{c}}$ over $10^{6}$ $\mathrm{A} / \mathrm{cm}^{2}$ at $77 \mathrm{~K}$. The films were implanted with $\mathrm{Al}, \mathrm{Ag}, \mathrm{Ca}$, $\mathrm{Ni}$, and $\mathrm{Si}$ ions at different projection energies and with a dose ranging from $10^{14}$ to $10^{16} / \mathrm{cm}^{2}$. The selection of a reactive ion is based on the negative heat of formation of its oxide [29]. Among the ions studied, Al is the most reactive with oxygen, followed by $\mathrm{Ca}, \mathrm{Si}$, and $\mathrm{Ni}$. In contrast, $\mathrm{Ag}$ is not reactive with oxygen and can not inhibit superconductivity in HTS oxides.

To investigate the chemical reaction between the implanted ions and HTS oxides, we performed X-ray photoelectron spectroscopy (XPS) measurements on a silicon implanted YBCO film. The results, plotted in figure 1, show

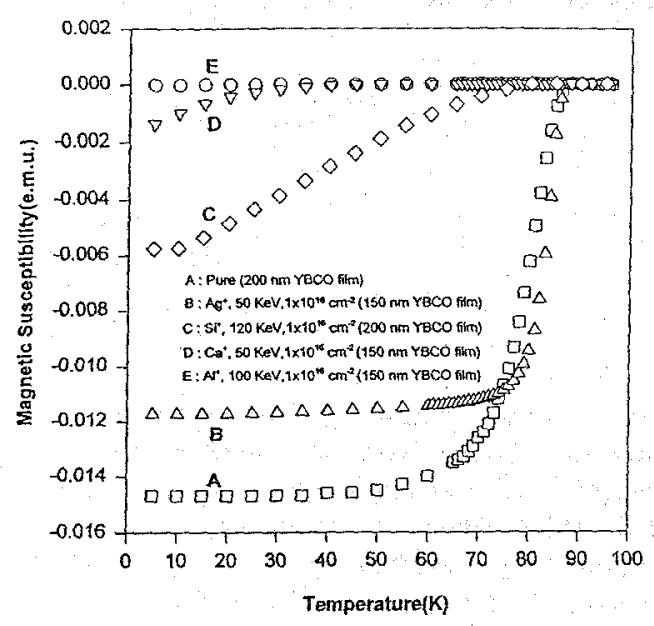

Fig. 2. Magnetic properties of implanted YBCO films with different energies and ions.

a chemical shift in binding energy of silicon $2 \mathrm{p}$ core-level from $99 \mathrm{eV}$ (that of bulk Si) to about $102 \mathrm{eV}$ (close to that of $\mathrm{SiO}_{2}$ ), indicating the formation of silicon oxides in the film. The inhibition of superconductivity is not due to the structural damage caused by implantation, rather it is mainly due to the formation of oxides after implantation. The X-ray diffraction measurements of implanted films do not show significant structural damage [20]. Further, under similar processing conditions, the reactive $\mathrm{Al}, \mathrm{Ca}$, and Si ions inhibit the diamagnetism of a YBCO film while the $\mathrm{Ag}$ ion has no effect on the Tc of the film. As an example, figure 2 shows the susceptibility measurements of YBCO films implanted with $\mathrm{Al}, \mathrm{Ca}, \mathrm{Si}$, and $\mathrm{Ag}$ at the same dose.

To inhibit superconductivity, the correct parameters in the implantation process have to be chosen, particularly the ion dose and projection energy. Figure 3 shows the susceptibility of A) a pure YBCO (2000 $\AA$ thick) film, B) the film implanted with $\mathrm{Ni}$ at $200 \mathrm{keV}$ and a dose of $1 \times 10^{13} / \mathrm{cm}^{2}, \mathrm{C}$ ) same as B) but at a dose of $5 \times 10^{13} / \mathrm{cm}^{2}$, and D) at a dose of $1 \times 10^{14} / \mathrm{cm}^{2}$. As one can see from the figure, the low dose sample still shows a diamagnetic transition. Increasing the $\mathrm{Ni}$ dose by one order of magnitude to $10^{14} / \mathrm{cm}^{2}$ completely inhibits the diamagnetism. The resistances of the $\mathrm{Ni}$ implanted films were measured as a function of temperature and plotted in figure 4 . It was found that the lower dose samples of $1-3 \times 10^{13} / \mathrm{cm}^{2}$ were still superconducting while the higher dose sample of $1 \times 10^{14} / \mathrm{cm}^{2}$ became semiconducting.

The selection of ion projection energy is even more critical than that of dose to ensure the inhibition. In general, the projection range should be in the middle of the film depth and the film thickness should be two to four times of the longitudinal straggling range. For a comparison study, we implanted Si ions into a $1100 \AA$ YBCO film at three differen energies of $5 \mathrm{keV}, 10 \mathrm{keV}$ and $80 \mathrm{keV}$. The distribution $\mathrm{o}$ the $\mathrm{Si}$ ions are measured by SIMS and the results are showi in figure 5 for implant energies of $5 \mathrm{keV}, 10 \mathrm{keV}$, and 81 $\mathrm{keV}$, respectively. From the figures, it is obvious that th ions with a low energy only reach the surface region of the 


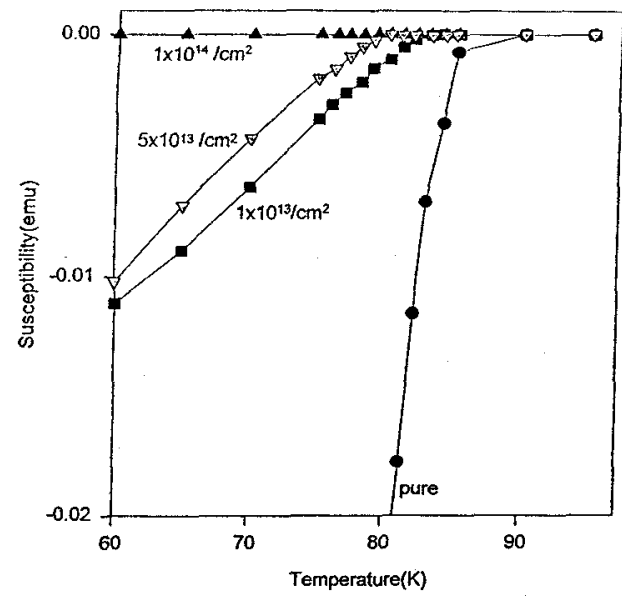

Fig. 3. Magnetic properties of Ni implanted YBCO films with different doses of $\mathrm{Ni}$ ions.

film. The Si ions at $80 \mathrm{keV}$ are centered at $600 \AA$ and its profile covered the entire film thickness. The results of the susceptibility measurement are plotted in figure 6 . The 5 $\mathrm{keV}$ and $10 \mathrm{keV}$ ions only penetrate into the film at the depth of $60 \AA$ and $100 \AA$. This leaves the major portion of the film and its superconducting properties unaffected, as shown in curves $\mathrm{A}$ ) and $\mathrm{B}$ ). To cover the entire film thickness, a 80 $\mathrm{keV}$ implant is used, which has a peak concentration at 600 $\AA$. Superconductivity is inhibited for a $80 \mathrm{keV} \mathrm{Si}$ implant, as shown in curves $C$ ) and $D$ ).

For a thick film $(>1500 \AA)$, a high energy implant has to be used in order for ions to cover the entire film thickness. Figure 7 shows the SIMS profiles of $\mathrm{Al}$ ions as well as the HTS elements. The $\mathrm{Al}$ ions were implanted at $100 \mathrm{keV}$ which distributed over a $2000 \AA$ film. It is noticed that the profiles of HTS elements are uniform throughout the film depth, indicating no stochiometric distortion in material due to the implantation.

To obtain a uniform distribution of the ions across the film, multiple implants should be used. Figure 8 shows the

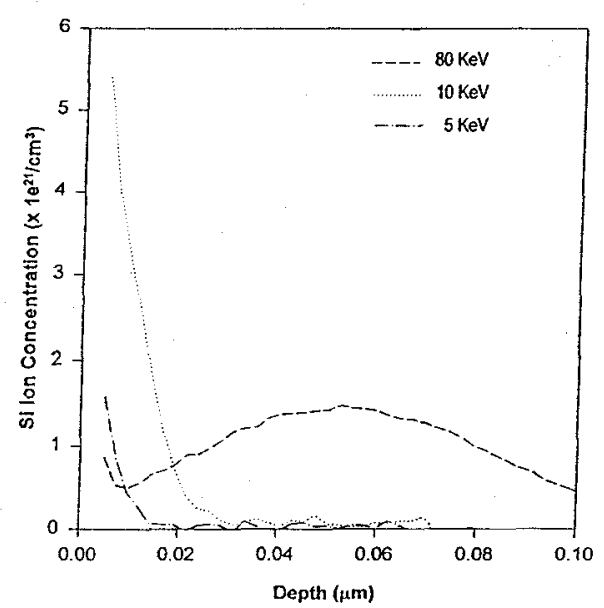

Fig. 5. Secondary ion mass spectroscopy(SIMS) depth profile of Si ions in YBCO film with different energies.

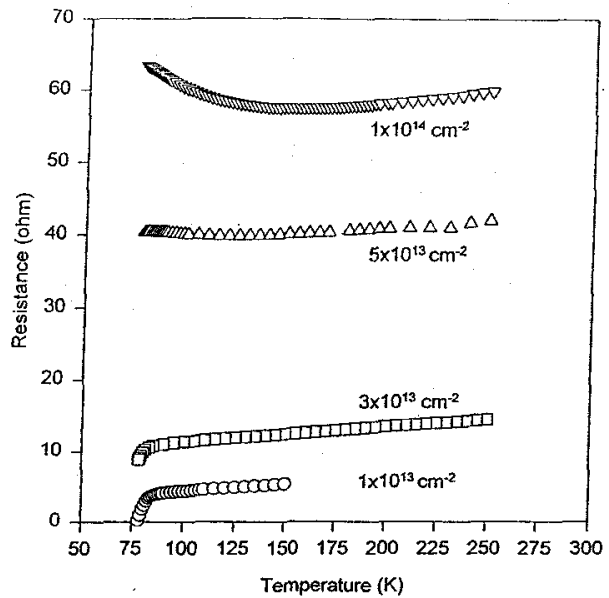

Fig.4. Resistance vs. Temperature curve for Ni implanted YBCO films with different doses of Ni ions.

SIMS data for Si implants at four different energies and doses. These parameters were determined by the TRIM simulation results, which are also plotted in the figure. The Si ions have almost a flat distribution throughout the film thickness. To study the effect of ion diffusion, a systematic annealing was carried out on Si implanted samples. In the temperature range of $300-600 \mathrm{C}$, the inhibition is enhanced.

Annealing of Si implantaed film samples at temperature higher than $600 \mathrm{C}$ leads to an interesting phenomenon. Instead of diffusion from a high concentration region to a low one, the $\mathrm{Si}$ ions move from a low concentration region to a high one where the crystal structural damage is severe [30]. This gettering effect creates a narrow inhibited layer within a HTS film and may be used to make superconductor-normalsuperconductor trilayer structure within a single film.

\section{DEVICE PRocessing}

The patterning of single-layer HTS films is carried out by implantation with a photoresist (PR) pattern. The PR is

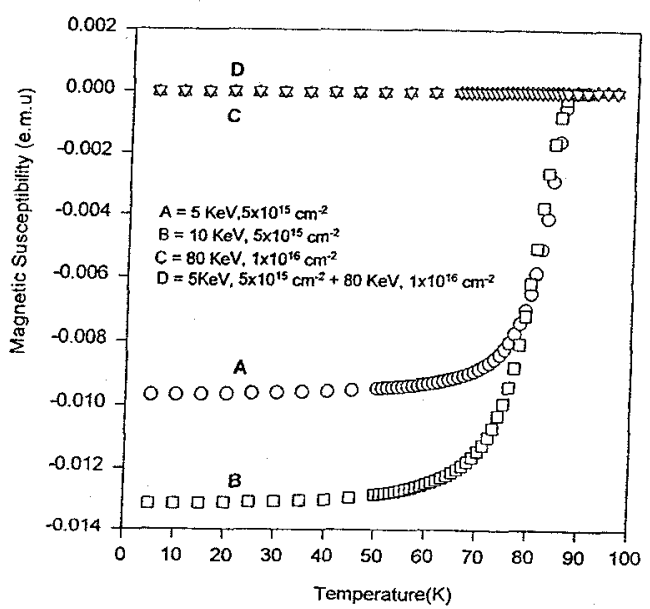

Fig. 6. Magneitc properties of Si implanted YBCO with different energies and doses. 


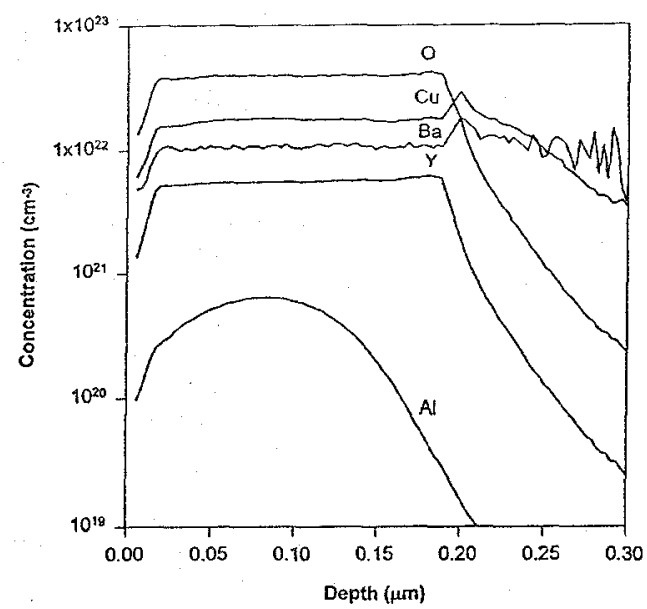

Fig. 7. Secondary ion mass spectroscopy(SIMS) depth profile of $\mathrm{Al}$ ion implanted YBCO film.

applied to a HTS film using standard photolithographic processes, and subsequently removed by acetone and oxygen plasma cleaning. For a high dose implantation, water cooling is used to avoid the formation of cracks in the PR during the implantation process.

For single layer device process, we have fabricated step edge junctions, DC SQUIDs [23], coplanar waveguides [24, 25], and RF coils [26-28] by the ion implantation process. Step edge junctions with $2 \mu \mathrm{m}$ line widths have been achieved and operational SQUIDs have been demonstrated at $77 \mathrm{~K}$ [23]. The legs of the SQUID loop containing the stepedge junctions are designed at $4 \times 8 \mu \mathrm{m}^{2}$ and the inner area of the loop is $40 \times 40 \mu \mathrm{m}^{2}$. The performance of the SQUID is plotted in Figure 9. This device showed a voltage modulation depth of $5 \mu \mathrm{V}$ at $77 \mathrm{~K}$, which is comparable to ion-milled SQUIDs.

Figure 10 shows the results of broadband attenuation constant measurements of three devices with the same coplanar waveguide (CPW) transmission line structure. The devices are fabricated by ion-milling, Si implantation, and $\mathrm{Al}$ implantation with the identical photolithograph masks. In

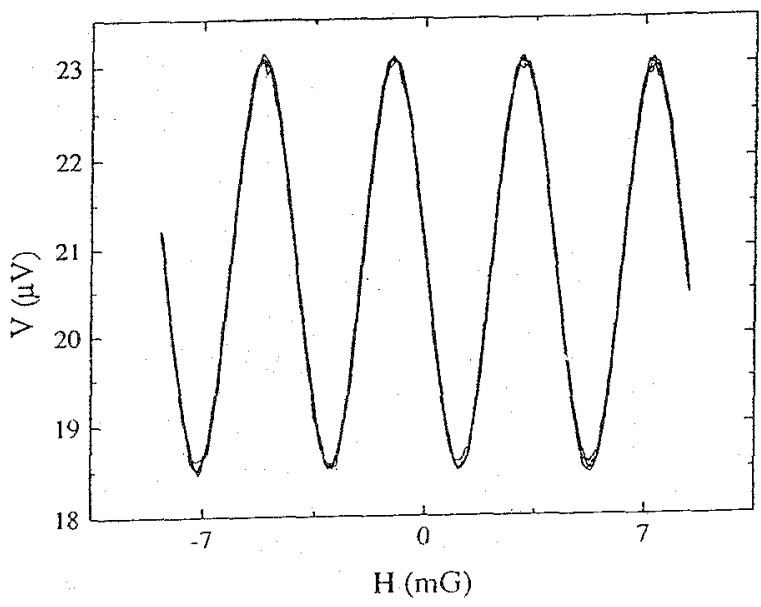

Fig. 9. The characteristic voltage versus magnetic field curve of the HTS DC SQUID at $77 \mathrm{~K}$.

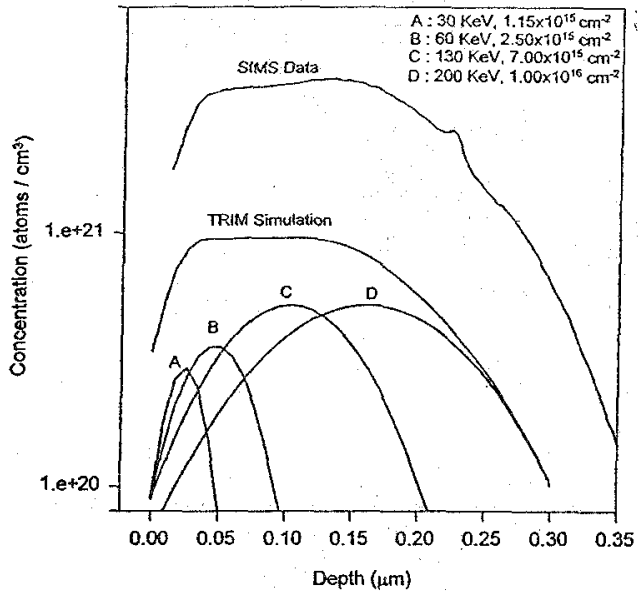

Fig. 8. Secondary ion mass spectroscopy(SIMS) depth profile and TRIM calculation of Si ions in YBCO with multiple implantation.

addition, the attenuation data derived from measurements of the quality factors (Q) of resonator made on the three samples are plotted in the figure. The $\mathrm{CPW}$ lines are characterized with resonant and broadband measurements to obtain accurate S-parameters and propagation constants from $1 \mathrm{GHz}$ to $25 \mathrm{GHz}[25]$. The propagation constants of ion: implanted lines are the same as those of lines patterned by conventional ion milling process, with a value for the attenuation constant of $0.03-0.04 \mathrm{~dB} / \mathrm{cm}$ at $10 \mathrm{GHz}$ and $50 \mathrm{~K}$. The lowest value of $0.027 \mathrm{~dB} / \mathrm{cm}$ is measured from $\mathrm{Si}$ implanted lines, which is lower than that of ion milled lines $(0.037 \mathrm{~dB} / \mathrm{cm})$. The $\mathrm{Al}$ implanted lines show the slightly higher value of $0.04 \mathrm{~dB} / \mathrm{cm}$. The relative dielectric constant of the CPW structures are also measured. The data shows that the phase constant is independent of frequency $(50 \mathrm{MHz}$ $25 \mathrm{GHz}$ ) with a value of 13 for both ion-implanted and ion milled lines. The relative low losses of ion implanted devices demonstrate the effectiveness of the RII technique for HTS microwave fabrication.

Figure 11. is a photo of a portion of a RF surface coil for magnetic resonance imaging (MRD). The coil is about 1 inch



Fig. 10. Attenuation values measured from broadband transmission and from resonator for all three samples. 


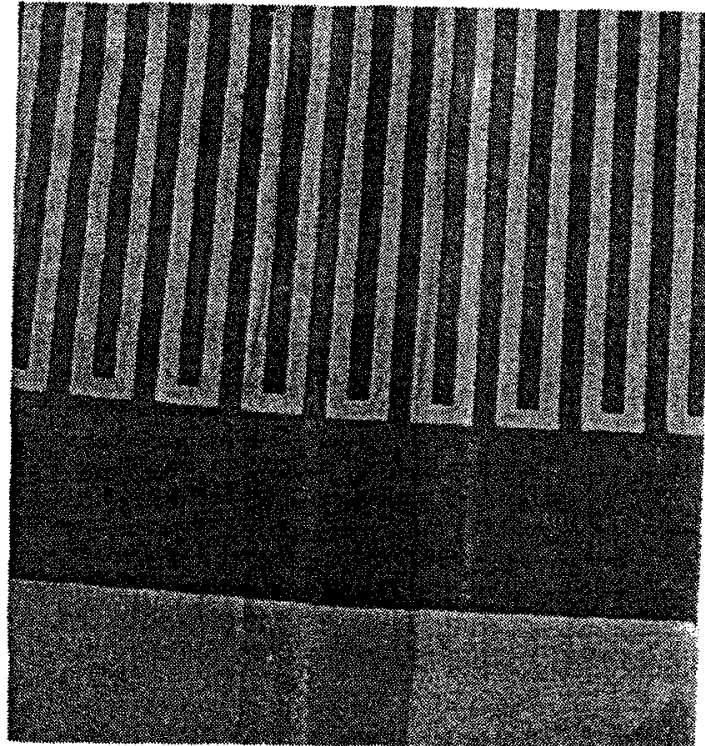

Fig. 11. A magnified photo showing the interdigitized capacitors of a HTS RF coil fabricated by ion implantation.

in diameter and consists of an single turn inductor with an interdigital capacitors [26]. The dark area is YBCO film and the light area is implanted with $\mathrm{Si}$. The line and spacing are about $20 \mu \mathrm{m}$ in width. The coils are designed and fabricated for $\mathrm{Na}$ and proton $(\mathrm{H}) \mathrm{MRI}$ at 3 Tesla with resonance frequencies of 33.7 and $127.7 \mathrm{Mhz}$, respectively. The quality factors of the HTS coil are about one to two-orders of magnitude higher than that of a conventional body coil made of $\mathrm{Cu}$. As an example, the Qs fro $\mathrm{Na}$ and $\mathrm{H}$ frequencies reach 7800 and 11090, respectively. Such high Qs improve the signal-to-noise ratio by a factor of 10 in both $\mathrm{Na}$ and $\mathrm{H}$ MRI $[27,28]$. Details of the coil characteristics are described elsewhere [26].

For multilayer device fabrication, we have made a simple $\mathrm{YBCO} / \mathrm{STO} / \mathrm{YBCO}$ trilayer device structure. The structure is illustrated in Figure 12 (top). The first YBCO layer is patterned by $\mathrm{Si}$ implantation. After removal of $\mathrm{PR}$, a $\mathrm{SrTiO}_{3}$ (STO) buffer layer and the second YBCO layer are deposited on the sample. A second $\mathrm{Si}$ implantation is carried out through a different PR mask.

Figure 12 (bottom) shows a photo of portion of a resonator with a coupled parallel plate capacitor. As seen from the photo, the inductor ring (light) has a open window (dark) in the first YBCO layer. The open area is implanted with Si. The patterned second YBCO layer, separated by a STO buffer layer, has a section overlapped with the first ring gray), which makes a parallel capacitor. Again, all three zyers remain planar. This tri-layer resonator is also designed nd fabricated for MRI in the frequency range of $30-120$ $1 \mathrm{~Hz}$. It is interesting to note that the ion implantation work HTS oxide can be easily applied to other oxides, such as electric, ferroelectric, and magnetoresistance materials.
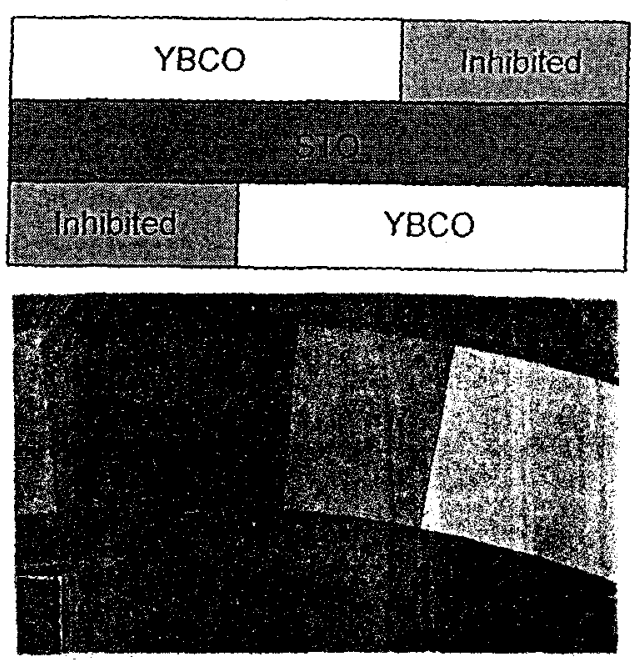

Fig. 12. A planar trilayer RF resonator with two implantations and two deposition steps.

Those oxides have the similar perovskite structure as HTS. Using ion implantation, we have doped YSZ oxide and increased conductivity by two orders of magnitude [31].

\section{CONCLUSION}

In conclusion, a planar inhibition device processing technique is presented. Material properties of ion-implanted HTS films are studied in detail. A variety of single-layer devices such as SQUIDs, RF coils, and waveguides have been demonstrated. This process offers two advantages: planar patterning for multilayer device and no chemical contamination to the patterned devices.

\section{ACKNOWLEDGMENT}

The author would like to thank S. Hong, J. Miller, K. Zhang, K. Viggiano, M. Chen, J.Baniecki, G.Luke, Y. F. Yang, E. S.Yang, M. Crow, I. Mun, P. Alderson, of Columbia University; D. Face, D. Kountz, of DuPont Superconductivity; S. Haupt, J. Sun, W. Gallagher, R. Laibowitz, of IBM; J. Lagraff, J. Murdock, H. Chan, of TRW; J. Booth, J. Beall, D. DeGroot, D. Rudman, and R. Ono of NIST and A. Wong, W. Hardy, H. Kato, N.A.F. Jaeger of UBC for their assistance with this work.

\section{REFERENCES}

[1] J. Clarke, "SQUIDs" Scientific American, Aug., pp. 46-53, 1994.

[2] R. B. Laibowitz, J. Z. Sun, V. Foglietti, W. I. Gallagher, and R. H. Koch "High-Tc multilevel edge junction superconductor quantum interference devices with $\mathrm{SrTiO}_{3}$ barriers operating at 77K", Appl. Phys. Lett, vol. 64, pp. 246, 1994. 
[3] L. P. Lee, K. Char, M. S. Colcough, and G. Zaharchuk "Monolithic $77 \mathrm{~K}$ dc SQUID magnetometer", Appl. Phys. Lett., yol. 59, pp.3051, 1991.

[4] M. J. Burns, K. Char, B. F. Cole, W. S. Rudy, and S. A. Sachtjen "Multichip module using multilayer $\mathrm{YBCO}$ interconnects", Appl Phys. Lett, vol. 62, pp. 1435, 1993.

[5] A. I. Braginski, "Thin Film Structures" in "The New Superconducting Electronics" edited by H. Weinstock and R. W. Ralston, NATO ASI Series, vol. E251, Kluwer Academis Pub., 1993.

[6] M. F. Yan, R. L Bams, H. M. O'Bryan, P. K. Gallagher, R. C. Sherwood, and $S$. Jin "Water interaction with the superconducting YBaCuO phase", Appl. Phys, Lett., vol. 51, pp. 532, 1987.

[7] R. P. Vasquez, "Chemical modification of HTS surfaces" in "Synthesis and Characterization of High Tc Superconductors" edited by J. J. Pouch, et al, Trans Tech Pub., Switzerland, 1993.

[8] F. C. Wellstood, J. J. Kingston, and J. Clarke "Thin-film multilayer interconnect technology for $\mathrm{YBaCuO}^{\prime}, J$. Appl. Phys., vol. 75, pp.683, 1994.

[9] F. C. Wellstood, J. J. Kingston, M. J. Ferrari, and J. Clarke "Superconducting thin film flux transformers of $\mathrm{YBaCuO}$ ", Appt. Phys. Lett., vol. 57, pp. 1930, 1990.

[10] Q. Y. Ma, G. V. Treyz, E. S. Yang and Chin-An Chang, "A novel method for patterning $\mathrm{YBaCuO}$ superconducting thin films", Appl. Phys. Lett., vol. 55, pp.896, 1989.

[11] Q. Y. Ma, E. S. Yang, R. B. Laibowitz, and Chin-An Chang "SiYBCO intermixing and reactive patterning technique", $J$. Elec. Mat., vol. 21, pp. $407,1992$.

[12] Q. Y. Ma and E. S. Yang, "Fabrication of HTS Electronic Devices With Reactive Patterning Technique." in "Synthesis and Characterization of High To Superconductors", edited by J. J. Pouch, et al, Trans Tech Pub. Switzerland, 1993.

[13] T. Hatano, A. Fuhimaki, Y. Takai, and H. Hayakawa, Jap. J. Appl. Phys., vol. 29, pp. 1076, 1990.

[14] Q. Y. Ma and E. S. Yang, "Current controlled high $T_{c}$ superconducting switch", Cryagenics, vol. 30, pp. 1146, 1990.

[15] D. P. Kern, K. Y. Lee, R. B. Laibowitz, and A. Gupta, "High resolution patterning of high Tc superconductors", J. Vac. Sci. Tech. vol. 89 , pp. $2875,1991$.

[16] C. Camerlingo, H. Huang, B. Ruggiero, M. Russo, E. Samelli, G. Testa, L. Correra, S. Guerri, S. Neri, S. Nicoletti, G. Pizzochero, Y. Su. "Step-edge junction fabrication by a Si/YBCO reactive patterning technique", Physica C., vo1. 232, pp. 44-48, 1994.

[17] G. Friedl, M. Vildic, B. Roas, D. Uhl, F. Bommel, M. Romheld, B. Hillenbrand, B. Stritzker, and G. Daalmans "Low 1/f noise singlelayer YBaCuO dc SQUID at 77K", Appl. Phys. Lett., vol. 60 , pp.3048,1992.
[18] B. E. Cole, SPIE, pp. 1394,1991, and P. W. Kruse, SPIE, pp. 1292, 1990.

[19] R. Parsons, G. Clarke, N. Osborne, Q. Y. Ma, F Orfino, L, N. Phong, W.Hardy, and S. Wessel, "Fabrication of High Te films on silicon membranes for bolometricdetectors" extended abs. of the "17th International Conf. on Infrared and Millimeter Waves" in Los Angeles, CA, Dec., 1992:

[20] Q. Y. Ma, P. Dosanjh, A. Wong, J. Carolan, and W. N. Hardy "Inhibition of conductivity in HTS film by Si ion implantation", Appl. Phys. Lett. vol. 63, pp.3633, 1993.

[21] Q. Y. Ma, A. Wong, P. Dosenjh, J. Carolan, W. Hardy", A planar method for patterning of HTS films and multilayers", Appl. Phys. Lett., vol. 65 , pp. $240,1994$.

[22] X. He, J. Musolf, E. Waffenschmidt, K. Heime, H. Wolf and K. Pier, "Silicon Ion Implantation of $\mathrm{YBaCuO}$ Films for Bolometer Application", IEEE Trans. Appl Supercond., vol. 5, pp. 2439, 1995.

[23] O.Y. Ma S. H. Hong, J R. Miller, J Sun, W. J Gallagher, and R. B. Laibowitz "High Tc SQUIDs fabricated by inhibiting ion implantation", Supercond Sci. \& Tech, vol. V9, pp. A92-95, 1996.

[24] D.C.DeGroot, D.A.Rudman, Kuan Zhang, Q.Y.Ma, H.Kato, N.A.F.Jaeger, "Planar microwave devices fabricated by ionimplantation patteming of high-temperature superconductors", $A p p l$. Phys.Left. vol.69, pp. 2119, 1996.

[25] J.C.Booth, J.A.Beall, D.C.Groot, D.A.Rudman, R.H.Ono, J.R.Miller, M.L.Chen, S.H.Hong, Q.Y.Ma, "Microwave Characterization of Coplanar Waveguide Transmission Lines Fabricated by Ion Implantation Patterning of $\mathrm{YBCO}^{\prime}$. (submitted to IEEE Trans. App. Sup.)

[26] J.R.Miller, K.Zhang, Q.Y.Ma, I.K.Mun, K.J.Jung, J.Katz, D.W.Face, D.J.Kountz, "Superconducting Receiver Coils for Sodium Magnetic Resonance Ithaging", LEEE Trans. on Biomedical Eng., vol. 43, pp. $1197,1996$.

[27] J. R. Miller, K. Zhang, Q. Y. Ma, I. K. Mun, K. J. Jung, J. Katz, D. W. Face, and D. J. Kountz "High Temperature Superconducting Receiver Coils for Sodium Imaging", Proc. Soc. Mag. Reson.1, pp. 255 1996.

[28] J.R.Miller, Q.Y.Ma, E.S.Yang, P.O.Alderson, I.K.Mun, Jose Katz, D.W.Face, D.J.Kountz., "A Multiple Nuclei Superconducting Probe for MRI", Ext. Abs. for Soc. Mag. Res. Meeting, 1997.

[29] CRC Handbook of Chemistry and Physics, 66th ed., 1985.

[30] S. H. Hong, M. L Chen, J. Baniecki, Q. Y. Ma, A. Wang, and R.W. Odom "Observation of ion gettering effect in YBCO oxides", $A p p l$. Phys. Lett., vol. 69, pp. 1629, 1996.

[31] S.H.Hong, J.R.Miller, Q.Y.Ma, E.S.Yang, D.B.Fenner, C.Y.Yang, J.I.Budnick, Mater. Res. Soc. Symp. Proc, vol. 401, pp. 309, 1996 